highly stable cytoskeleton, the level of this protein is downregulated about 1,000 times in an actin-independent manner ${ }^{8}$. Gelsolin appears to play an important role in these major cytoskeletal changes, but is it unique?

Actin is promiscuous in cells: it can interact with several partners at once. The choice depends on the relative binding affinities and cellular concentrations of the different proteins and on regulatory factors. Because phenotypic changes may depend on the balance of different actinbinding proteins, it is important to assess changes in several of these proteins simultaneously. The levels of gelsolin, profilin, filamin and actin itself have been measured during differentiation of two myeloid cell lines as they acquire the phagocytic and locomotory properties of macrophages. Gelsolin mRNA levels were found to increase 5-10-fold over two days with a corresponding 40-50-fold increase in protein levels, whereas filamin mRNA increased only 2-4-fold with a corresponding 3-7-fold increase in protein levels and there was a greater than twofold increase in mRNA or protein levels of profilin or actin. The levels of gelsolin, filamin and actin in the differentiated cell lines were similar to those of rabbit macrophages. By contrast, a leukaemic cell line, which differentiates only slightly, showed insignificant changes in the levels of any of these proteins.

These observations emphasize the importance of gelsolin in dynamic rearrangements of the actin cytoskeleton during the spreading and subsequent locomotion of cells, but changes in the levels of other actin-binding proteins may be no less important. Melanoma cell lines lacking filamin show apparently uncontrolled surface blebbing activity together with loss of locomotive capacity ${ }^{10}$. Like a car with the clutch disengaged, the engine races but no movement results.

Although rearrangements of cytoskeletal architecture can be correlated with the expression of various actin-binding proteins, this does not prove a causative role. Functional requirements have been explored by disrupting the expression of individual actin-binding proteins. This approach has been particularly successful for myosin using Dictyostelium discoideum, an amoeboid cell showing well-defined locomotory behaviour and a developmental cycle. Clear evidence has emerged for the importance of myosin II (analogous to skeletal muscle myosin) in cytokinesis $^{11}$ and the capping of surface receptors in these cells ${ }^{12}$, but cells devoid of this myosin are still capable of cytoplasmic organelle movement, chemotaxis and locomotion. The single-headed globular myosin, myosin I, which does not form filaments and is concentrated at the leading edges of amoebae in motion, may be responsible for these motile activities ${ }^{13}$.

\title{
Sticklebacks on red alert
}

WHAT is the advantage to a female of being choosy about her mating partner? Of the several possible answers to this question, one in particular receives support from the work of M. Milinski and T. C. M. Bakker described on page 330 of this issue.

One explanation of sexual selection by female choice is that the male traits preferred are arbitrary, in the sense that they the results of a study demonstrating that, in the three-spined stickleback Gasterosteus aculeatus, females prefer males with the brightest red on their bellies (a trait that is very apparent in the males of the species when in breeding condition, as the picture shows) and that this preference may have evolved as a way of avoiding parasitized males. It seems, perhaps not surprisingly,

\section{IMAGE UNAVAILABLE FOR COPYRIGHT REASONS}

Love-nest - a male three-spined stickleback Gasterosteus aculeatus inducing a female to spawn in the nest he has built for the purpose.

confer no direct fitness benefit on offspring, and that they have evolved along with the female mating preference by a kind of 'runaway process'. According to this idea, first suggested by the geneticist R. A. Fisher, the male offspring of preferential matings tend to inherit their fathers' attractive traits, in turn making them more attractive to females when they reach maturity. Female offspring, on the other hand, would tend to inherit their mothers' preferences, the two processes acting together to build up a genetic correlation between preference and preferred trait.

Alternatively, female preferences for particular traits may have come about because of nonadaptive (or even maladaptive) correlates of the otherwise adaptive wiring of the brain. For an example where this may have occurred, see M. J. Ryan et al.'s study of sexual selection by 'sensory exploitation' in the tungara frog (Nature 343, 66-67; 1990), the conclusion of which is that sensory bias unrelated to any 'runaway process' may be responsible for the female preference to particular male courtship sounds, or 'chucks'.

It is a third explanation - that the traits preferred by females serve to advertise the superior 'quality' of the males, where the 'quality' may be a heritable fitness component - that the report by Milinski and Bakker bears upon. The authors describe that in this species the intensity of the red breeding coloration correlates with the physical condition of the males, and that males infected by the ciliate parasite that causes 'white spot' suffer a decrease in the intensity of their coloration leading to a reduction in their attractiveness to females. The female preference for brighter, redder males apparent in white light breaks down in green light; together with the results of other experiments, this observation leads the authors to the conclusion that it is the red coloration, and not the presence or absence of parasites as such, that is the cue used when selecting mates.

As Milinski and Bakker readily admit, their study ignores many important aspects of courtship and mating in the three-spined stickleback and is not the last word on female choice in this species. It is not yet known whether in the wild males with brighter coloration tend to be those that are less parasitized or whether coloration is a reflection of heritable differences between males. Furthermore, in this species males care for the eggs and fry until several days after spawning. Therefore, females may avoid parasitized males not because of any genetic benefit that might accrue to their offspring, but because such males may be less capable of paternal care than healthier males.

Rory Howlett 